\title{
ANSIEDAD Y CÓLERA EN ESTUDIANTES DE LAS CIUDADES DE LIMA Y TRUJILLO CON PARTICIPACIÓN Y NO PARTICIPACIÓN EN ACTOS VIOLENTOS
}

\author{
Carlos Velásquez Centeno \\ Carlos Arenas Iparraguirre \\ Manuel Campos Roldán \\ Alejandro Dioses Chocano \\ Víctor Gutiérrez Olaya \\ Luis García Vega \\ Roger Elizalde Bravo \\ Víctor Montero López
}

\begin{abstract}
RESUMEN
Se resalta que en la problemática del recrudecimiento de la violencia cotidiana tienen mucho que ver los estados disposicionales de la ansiedad, la cólera y la hostilidad con relación a ciertos factores estructurales y coyunturales injustos, por ello cualquier intervención psicológica con pretensiones efectivas tendrá que basarse en el conocimiento a fondo de los mecanismos de tales fenómenos. En cuanto a los factores disposicionales extremos, ellos han sido ignorados en relación con la violencia, por lo cual la presente investigación plantea una aproximación evaluativa a nivel de juventud escolar de Lima y Trujillo, utilizando dos instrumentos: el Inventario de Ansiedad Rasgo-Estado y el Inventario Multicultural de cóleraHostilidad, mediante los cuales se analiza una muestra conformada por alumnos de 19 colegios de centros educativos nacionales. Los resultados indican una relación baja y muy baja entre la Ansiedad y la Cólera en los alumnos que participan y no participan en actos violentos; mientras que por otra parte existen correlaciones moderadas y diferencias significativas en la expresión de la Cólera hostilidad entre los estudiantes de Lima y Trujillo siendo mayor en gran parte en los que participan en actos violentos.
\end{abstract}

Palabras Clave: Estados disposicionales emotivos, ansiedad, rasgo-estado, cólera, hostilidad, violencia juvenil y cotidiana, instrumentos de evaluación.

\begin{abstract}
You points that in the problem of the worsening of the daily violence they have a lot to do at the disposals states of the anxiety, the anger and the hostility with relationship to certain unjust structural and of the situation factors, for it any psychological intervention with effective presumptions will have to thoroughly be based on the knowledge of the mechanisms of such phenomenons. As for the factors disposals extreme, they have been ignored in connection with the violence, reason why the present investigation outlines an evaluative approach to school youth 's from Lima level and Trujillo, using two instruments: the Inventory of Anxiety Feature-state and the Multicultural Inventory of cholera-hostility, by means of which a sample is analyzed conformed by students of 19 state schools. The results indicate a low and a lowest relationships between the Anxiety and the Anger in the students that participate and they don't participate in violent acts; while on the other hand exist a moderate correlation and significative differs in the expression of the Anger hostility between the students from Lima and Trujillo being bigger largely in those that participate in violent acts.
\end{abstract}

Words Key: disposals states moving anxiety feature-state, cholera, hostility, juvenile and daily violence, evaluation instruments.

Colaboradores de Lima: Montgomery W; Biminchumo L.; Roca J.; Allen S..; Vallejo J.; Dextre M.; Guevara W; Amaya J. y Girau J. Colaboradores de Trujillo : Vela O.; Reyes G.; Alarcón R. y Asmat C.. 
La violencia cotidiana es un fenómeno con distintas manifestaciones: agresión física y psicológica, abandono moral y material, abuso sexual, etc.; expresadas a través de las golpizas, el maltrato infantil y de mujeres, las torturas y quemaduras, la calumnia, los crímenes, violaciones, secuestros, insultos y otras modalidades. Su ocurrencia tiene por víctimas a gente de cualquier sexo y edad, obstaculizando su desarrollo personal y provocando daños frecuentemente irremediables.

Un estudio epidemiológico reciente sobre la violencia en Lima y Callao (Anicama, Vizcardo, Carrasco y Mayorga, 1999) revela interesantes datos acerca de ella. Por ejemplo, respecto al área familiar se encuentra que un $28 \%$ de los individuos encuestados recibe asiduamente insultos o agresiones de su pareja; por otro lado hay un $54 \%$ de la muestra que declara castigar físicamente a sus hijos; $y$, en el área de violencia callejera, un $44 \%$ indica haber sido víctimas de algún acto delincuencial. En cuanto al papel protector del Estado (seguridad urbana), un 68\% de respuestas desaprobatorias da idea de la desconfianza de los ciudadanos hacia él.

Lo cierto es que el recrudecimiento de la violencia es el Perú viene concitando preocupación general y un verdadero alud de investigaciones sobre su alcance, desde las que se podrían considerar las predominantemente académicas (Moragues, 1982; Tong, 1995; Perales y Sogi, 1995; Orellana y García, 1995; Elizalde et al, 1997; Cruzado, 1997; Arenas, 1998; Montgomery, 1998; Velásquez, 1999), hasta las desarrolladas por organismos oficiales del Estado (Congreso de la República, 1998). En suma, tales estudios coinciden substancialmente en que la violencia es producida por dos grandes conjuntos de factores externos:

a) Una realidad estructural plagada de desigualdades, falta de oportunidades, falta de lugares y momentos de distensión, marginalidad, consumo de sustancias psicoactivas, irrespeto por los derechos humanos, pautas culturales inadecuadas, prejuicios y taras ideológicas perpetuadas por los medios dc comunicación social.

b) Familias y entornos con patrones de conducta agresiva y autoritaria por un lado, o excesivamente permisiva por otro (desfavoreciendo el aprendizaje de la disciplina y el respeto a las normas).

Las distintas manifestaciones y tipos de violencia han sido abordados abundantemente por reuniones de expertos convocados al efecto en eventos especiales (por ejemplo el de MacGregor, Rubio y Vega, 1990), así que aquí no se entrará en tales detalles. Lo que interesa sobre todo a la presente investigación es un aspecto colateral a semejante fenómeno, vinculado a un hecho fácilmente constatable: la mayoría de víctimas y victimarios de la violencia se hallan ente los 18 y 24 años de edad, como lo muestran los datos del Instituto Nacional de Estadística e Informática (INEI) hasta 1998. Asimismo, la población comprendida en esa etapa de la vida constituye un 55\% del total. Ello hace necesario fijar la atención en el segmento juvenil como un sector social en fuerte situación de riesgo.

Siendo así el asunto, es legítimo preguntarse hasta qué punto la serie de factores externos que propician situaciones de violencia impactan en el comportamiento emocional de los jóvenes peruanos. Como lo señala Ugarriza (1998), tal fenómeno ha sido a nivel de evaluación largamente ignorado, en la presunción de que eran otros factores (concretamente las diferencias individuales) los que influían de manera primordial. 
La ansiedad y la cólera son dos de las respuestas emocionales más notorias en dicho interjuego, formando una especie de «sedimento» conductual propicio para recibir y perpetuar actos de violencia en todos los ámbitos. El conocimiento de la manera como aparecen, se desarrollan y afectan el comportamiento situacional los estados disposicionales del tipo de la ansiedad y la cólera, permitirá llevar mejor el proceso de prevenir, predecir y controlar su incidencia en la población, especialmente en la juventud escolar. Tanto la ansiedad como la cólera son factores disposicionales que afectan la emisión, fuerza y textura de las conductas respondientes y operantes, e incluso de otros repertorios disposicionales. Por eso se les relaciona con diferentes variables y condiciones.

En cuanto a la ansiedad, por ejemplo García (1983) hizo un estudio probatorio de sus consecuencias debilitantes sobre el rendimiento escolar, especialmente en situaciones de índole evaluativo (véase también Quintana, 1998). El Inventario de ansiedad Estado-Rasgo elaborado en español por Spielberger y Díaz-Guerrero (1975), ha servido en numerosas oportunidades para medir esos estados en dichas contingencias (Bavermeister, 1986), y recientemente Inga (1999) también lo utilizó en el ámbito universitario para relacionar la ansiedad con el nivel de autoestima.

En lo que respecta a la cólera, su papel "directivo" en la producción de respuestas hostiles abiertas o encubiertas, así como sus correlatos a nivel patogénico, es bien conocido en los estudios sobre agresión (Bending, 1962) y medicina conductual (Crane, 1981).

La presente investigación pretende, pues, bordar con rigor científico el problema de la violencia juvenil, ampliando su marco teórico en aspectos colaterales de tipo disposicional, (ansiedad y cólera) desarrollando instrumentos válidos de medición psicológica para los profesionales de la salud, y constituyendo un referente para ajustar programas preventivos y de intervención a nivel nacional, tanto respecto a propuestas pasadas (ver Vallejos y Montero, 1990; Varona, 1991; Masías, 1994; Anicama, et al, 1997) como para las futuras.

Tales instrumentos ya existen y han demostrado ser adecuados en otras realidades, como el Inventario de Ansiedad Rasgo-Estado (IDARE) construido y sucesivamente refinado por Spielberger (1983; con Díaz-Guerrero, 1975) en numerosos estudios de investigación, desarrollando normas para estudiantes de educación secundaria y superior, empleados, personal militar, presos y pacientes hospitalizados. Otro instrumento relevante es el Inventario Multicultural de la Expresión de Cólera-Hostilidad (IMECH) de Moscoso y Reheiser (1996), recientemente adaptado por Ugarriza (1998) a nuestra realidad, y a nivel latinoamericano por el mismo Moscoso (1999). En dicha prueba el concepto de cólera refiere un estado emocional que va desde una leve irritación hasta una furia intensa, mientras que el de "hostilidad connota un conjunto de actitudes complejas que motivan conductas agresivas o violentas hacia personas u objetos.

En base a lo dicho, los objetivos de este trabajo se formulan así:

1) Establecer las relaciones entre la Ansiedad y la Cólera en función a la participación o no participación en actos violentos en escolares de las ciudades de Lima y Trujillo.

2) Realizar un análisis psicométrico del Inventario Multicultural de la Expresión de Cólera-Hostilidad y del Inventario de Ansiedad Estado-Rasgo en escolares de educación secundaria de Lima y Trujillo. 
3) Describir las características de la cólera - hostilidad y la ansiedad rasgo estado en escolares de educación secundaria que participan y no participan en actos violentos de la ciudades de Lima y Trujillo.

4) Determinar la validez, confiabilidad y normas de interpretación de ambos inventarios en escolares de educación secundaria de las ciudades de Lima y Trujillo.

Igualmente a nivel de hipótesis, fundamentado en las características de los estudios que respaldan los instrumentos señalados, se parte del supuesto que: a) los alumnos que participan en actos violentos de la ciudad de Lima y Trujillo presentan mayores niveles de ansiedad y cólera en comparación con los alumnos que no participan; y b) los alumnos de la ciudad de Lima que participan en actos violentos presentan mayores niveles de ansiedad y cólera que sus similares en la ciudad de Trujillo.

\section{MÉTODO}

\section{Muestra.}

Es una muestra intencional estratificada, que fue conformada por alumnos del 3ro al 5to de secundaria de los Centros Educativos Nacionales donde se detectaron actos de violencia estudiantil en las Ciudades de Lima y Trujillo, tal como figuran en el esquema siguiente:

\begin{tabular}{|c|c|c|}
\hline \multirow{\Xi}{\Xi}{} & \multicolumn{2}{|c|}{ Alumnos } \\
\multirow{\Xi}{\Xi}{} & $\begin{array}{c}\text { Sin participación } \\
\text { en actos violen los }\end{array}$ & $\begin{array}{c}\text { Con participación } \\
\text { en actos violento }\end{array}$ \\
\hline Lima & 556 & 516 \\
\hline Trujillo & 223 & 215 \\
\hline
\end{tabular}

\section{Instrumento}

Se obtuvo la validez, confiabilidad y baremación según las ciudades y condiciones de los estudiantes de participación y no participación en actos violentos del Inventario de Auto evaluación de la Ansiedad de Rasgo-Estado IDARE (Anexos $\mathrm{N}^{\circ} 1$ y 2) y del Inventario Multicultural de la Expresión de Cólera-Hostilidad IMECH (Anexos $\mathrm{N}^{\circ} 3$ y 4).

\section{Procedimiento.}

La muestra se conformó por alumnos de 19 Centros Educativos Nacionales de Lima Metropolitana y 8 Centros Educativos Nacionales de Trujillo, seleccionados por conocerse la existencia de actos de pandillaje, estableciéndose contacto a través de la Oficina de Coordinación Universitaria del Ministerio de Educación. La administración del IDARE e IMECH a los alumnos de la muestra intencional se realizó estableciendo coordinaciones con los Psicólogos Promotores del «Programa de Prevención de Violencia» de cada Centro Educativo de la Ciudad de Lima y con los Directores en la Ciudad de Trujillo, quienes sobre la base del conocimiento de su realidad agruparon a los alumnos de secundaria en dos grupos, de acuerdo a la variable participación o no participación en actos violentos. 


\section{Resultados}

TABLA No 1

MATRIZ DE CORRELACIÓN ENTRE LA ANSIEDAD Y LA COLERA EN ESTUDIANTES CON Y SIN PARTICIPACIÓN EN ACTOS VIOLENTOS DE LIMA Y TRUJILLO

\begin{tabular}{|c|c|c|c|c|c|c|c|}
\hline & A_RASGO & COL_ESTA & COL_RASG & COL_MAN & COL_CON & CONT_MAN & CONT_CON \\
\hline A_ESTADO &, $3542^{* * * *}$ &, $1615^{* * *}$ &, $0889 * * *$ &, $1168 * * *$ &, $1049 * * *$ &, $1728^{* * * *}$ &, 1007 \\
A_RASGO & &, $1876^{* * *}$ &, $3114 * * *$ &, $2144 * * *$ &, $2810^{* * *}$ &, $1611^{* * * *}$ &, $0844 * *$ \\
COL_ESTA & & &, $4358^{* * * *}$ &, $4178^{* * *}$ &, $3551^{* * *}$ &,$- 1975 * * *$ &,$- 2869 * * *$ \\
COL_RASG & & & &, $5235 * * *$ &, $5037 * * *$ &,$- 1662 * * *$ &,$- 2869 * * *$ \\
COL_MAN & & & & & & $-1,884 * * *$ &,$- 3361 * * *$ \\
\hline COL_CON & & & & & &,- 0493 &,$- 1526 * *$ \\
\hline CONT_MAN & & & & & & &,$- 5501 * * *$ \\
\hline
\end{tabular}

$*^{*} p<0,01$

$* * * p<0,001$

$N=732$

TABLA $\mathbf{N}^{\circ} 2$

MATRIZ DE CORRELACIÓN ENTRE LA ANSIEDAD Y LA COLERA EN ALUMNOS CON PARTICIPACIÓN EN ACTOS VIOLENTOS DE LAS CIUDADES DE LIMA Y TRUJILLO

\begin{tabular}{|c|c|c|c|c|c|c|c|}
\hline & A_RASGO & COL_ESTA & COL_RASG & COL_MAN & COL_CON & CONT_MAN & CONT_CON \\
\hline A_ESTADO &, $3890 * * *$ & ,2042*** &, $1248 * *$ &, $1514 * * *$ &, $1333 * * *$ &, $1918 * * *$ &, $0967 * *$ \\
\hline A_RASGO & &, $1757^{* * *}$ &, $2938 * * *$ &, $1873 * * *$ &, $2812 * * *$ &, $2017 * * *$ & ,1139** \\
\hline COL_ESTA & & & ,4281*** &, $3844 * * *$ &, $3486 * * *$ &,$- 1423 * * *$ &,$- 2037 * * *$ \\
\hline COL_RASG & & & &, $5338 * * *$ & ,4991*** &,$- 1422 * * *$ &,$- 2690 * * *$ \\
\hline COL_MAN & & & & &, $5338 * * *$ &,$- 1312 * * *$ &,$- 2810 * * *$ \\
\hline CONT_CON & & & & & &,- 0038 &,$- 1139 * * *$ \\
\hline CONT_MAN & & & & & & &, $5430 * * *$ \\
\hline
\end{tabular}


TABLA No 3

MATRIZ DE CORRELACIÓN ENTRE LA ANSIEDAD Y LA COLERA EN ALUMNOS SIN PARTICIPACIÓN EN ACTOS VIOLENTOS DE LA CIUDADES DE LIMA Y TRUJILLO

\begin{tabular}{|c|c|c|c|c|c|c|c|}
\hline & A_RASGO & COL_ESTA & COL_RASG & COL_MAN & COL_CON & CONT_MAN & CONT_CON \\
\hline A_ESTADO &, $3214 * * *$ &, $1182 * *$ & 0.055 & ,0852* &, $0775^{*}$ &, $1566 * * *$ &, $1056 * *$ \\
\hline A_RASGO & & ,2181*** & ,3360*** & ,2490**** & ,2844*** &, $1152 * *$ & ,0498 \\
\hline COL_ESTA & & & ,4380*** & ,4444*** &, $3620 * * *$ &,$- 2383 * * *$ &,$- 2618 * * *$ \\
\hline COL_RASG & & & &, $5086 * * *$ &, $5066 * * *$ &,$- 1787 * * *$ &,$- 2939 * * *$ \\
\hline COL_MAN & & & & & ,3840*** &,$- 2157 * * *$ &,$- 3738 * * *$ \\
\hline COL_CON & & & & & &,$- 0881 *$ &,$- 1834 * * *$ \\
\hline CONT_MAN & & & & & & &, $5482 * * *$ \\
\hline \multicolumn{8}{|l|}{$* \mathrm{p}<0,05$} \\
\hline \multicolumn{8}{|l|}{$* * \mathrm{p}<0,01$} \\
\hline \multicolumn{8}{|l|}{$* * * \mathrm{p}<0,001$} \\
\hline $\mathrm{N}=779$ & & & & & & & \\
\hline
\end{tabular}

TABLA N $\mathbf{N}^{\circ}$

PRUEBA DE MEDIAS EN LA QUE SE COMPARA LAS SUB-AREAS DEL IDARE Y EL IMECH EN ALUMNOS CON Y SOM PARTICIPACIÓN EN ACTOS VIOLENTOS DE LAS CIUDADES DE LIMA Y TRUJILLO

\begin{tabular}{|c|l|c|c|c|}
\hline \multirow{2}{*}{ PRUEBA } & \multirow{2}{*}{ AREA } & \multicolumn{2}{|c|}{ MEDIAS } & \multirow{2}{*}{ T } \\
\cline { 3 - 4 } & \multicolumn{2}{|c|}{ NO VIOLENTOS VIOLENTOS } & \\
\hline \multirow{3}{*}{ IDARE } & ANSIEDAD ESTADO & 43.11 & 43.12 &,- 058 \\
& ANSIEDAD Y RASGO & 47.22 & 46.71 & 1,61 \\
\hline \multirow{3}{*}{ IMECH } & CÓLERA ESTADO & 13.09 & 14.52 & $-5,29 * * *$ \\
& CÓLERA RASGO & 21.09 & 21.93 & $, 2,65 * * *$ \\
& CÓLERA MANIFIESTA & 10.79 & 11.32 & $-3,32 * * *$ \\
& CÓLERA CONTENIDA & 11.42 & 11.64 & $-1,28$ \\
& C. CÓLERA MANIFIESTA & 16.26 & 15.48 & $-3,74 * * *$ \\
& C. CÓLERA CONTENIDA & 15.8 & 14.95 & $4,14 * * *$ \\
\hline
\end{tabular}

$* * \mathrm{p}<0,01$

$* * * \mathrm{p}<0,001$ 
TABLA N 5

PRUEBA DE MEDIAS EN LA QUE SE COMPARA LAS SUB-AREAS DEL IDARE Y EL IMECH EN ALUMNOS CON Y SIN PARTICIPACIÓN EN ACTOS VIOLENTOS DE LAS CIUDADES DE LIMA

\begin{tabular}{|c|c|c|c|c|}
\hline \multirow{2}{*}{ PRUEBA } & \multirow{2}{*}{ AREA } & \multicolumn{2}{|c|}{ CONDICIÓN } & \multirow{2}{*}{$\mathbf{T}$} \\
\hline & & NO VIOLENTOS & VIOLENTOS & \\
\hline \multirow{2}{*}{ IDARE } & ANSIEDAD ESTADO & 43.07 & 42.96 &,- 31 \\
\hline & ANSIEDAD Y RASGO & 47.17 & 46.28 & 2,32 \\
\hline \multirow{6}{*}{ IMECH } & CÓLERA ESTADO & 13.31 & 14.41 & $-3,37 * *$ \\
\hline & CÓLERA RASGO & 21.13 & 21.34 &,- 59 \\
\hline & CÓLERA MANIFIESTA & 10.69 & 11.17 & $-2,61^{* *}$ \\
\hline & CÓLERA CONTENIDA & 11.47 & 11.44 &, $17^{*}$ \\
\hline & C. CÓLERA MANIFIESTA & 16.07 & 15.69 & 1,52 \\
\hline & C. CÓLERA CONTENIDA & 15.61 & 15.04 & $2,32 *$ \\
\hline
\end{tabular}

$* \mathrm{p}<0,01$

$* * * \mathrm{p}<0,001$

TABLA N ${ }^{\circ} 6$

PRUEBA DE MEDIAS EN LA QUE SE COMPARA LAS SUB-AREAS DEL IDARE Y EL IMECH EN ALUMNOS CON Y SIN PARTICIPACIÓN EN ACTOS VIOLENTOS DE LAS CIUDADES DE TRUJILLO

\begin{tabular}{|c|c|c|c|c|c|}
\hline \multirow{2}{*}{ PRUEBA } & \multirow{2}{*}{ AREA } & \multicolumn{2}{|c|}{ CONDICIÓN } & \multirow{2}{*}{$\mathbf{T}$} & \multirow{2}{*}{$\mathbf{P}$} \\
\hline & & NO VIOLENTOS & VIOLENTOS & & \\
\hline \multirow{2}{*}{ IDARE } & ANSIEDAD ESTADO & 43.18 & 43.52 &,- 62 &, 534 \\
\hline & ANSIEDAD Y RASGO & 47.34 & 47.74 &,- 77 & ,441 \\
\hline \multirow{6}{*}{$\mathrm{IMECH}$} & CÓLERA ESTADO & 12.55 & 14.78 & $-4,72 * * *$ &, $000 *$ \\
\hline & CÓLERA RASGO & 21 & 23.33 & $-3,87 * * *$ &, $000 *$ \\
\hline & CÓLERA MANIFIESTA & 11.02 & 11.68 & $-2,03^{*}$ &, $043^{*}$ \\
\hline & CÓLERA CONTENIDA & 11.3 & 12.14 & $-2,65 * *$ &, $008 *$ \\
\hline & C. CÓLERA MANIFIESTA & 16.73 & 14.96 &, $463 * * *$ &, $000 *$ \\
\hline & C. CÓLERA CONTENIDA & 16.27 & 14.72 & $4,11 * * *$ &, $000 *$ \\
\hline \multicolumn{6}{|l|}{$* p<0,05$} \\
\hline
\end{tabular}




\section{DISCUSIÓN}

En forma general (Tabla $\mathrm{N}^{\circ}$ 1) $\mathrm{Y}$ en las que se agrupan a los alumnos con y sin participación en actos violentos (Tablas $\mathrm{N}^{\circ} 2$ y 3), existe una correlación baja o muy baja entre la Ansiedad (AE-AR) y la Cólera (CE-CR).

Sin embargo, tanto los alumnos con y sin participación en actos violentos de Lima y Trujillo presentan una correlación moderada entre la cólera estado y la cólera rasgo, es decir entre el estar con cólera y sin cólera. Sin embargo los que no participan en actos violentos presentan una correlación moderada entre cólera estado y cólera manifiesta, significando esto que cuanto mayor es el sentimiento de estar con cólera este se dirige hacia personas u objetos del medio ambiente.

De la misma manera tanto en los alumnos con y sin participación en actos violentos muestran correlaciones moderadas entre la cólera rasgo con la cólera manifiesta y la controlada, indicando esto que se consideran coléricos y en algunas oportunidades expresan su cólera y en otra la contienen.

Por otro lado los alumnos violentos muestran correlaciones moderadas entre la cólera manifiesta y la controlada, indicándonos esto que cuando mayor es la expresión de la cólera de manera verbal o a través de una conducta agresiva, mayor es la supresión o control de sus sentimientos de cólera.

Por ultimo tanto los alumnos con y sin participación en actos violentos de Lima y Trujillo expresan la cólera en forma conductual y piensan en ella.

En relación a las diferencias entre las medias de los grupos de alumnos con participación y no participación en actos violentos correspondientes a la ciudad de Lima y Trujillo, se encuentra que hay deficiencias significativas en relación a la cólera-estado. cólera-rasgo y la cólera manifiesta. Del mismo modo se presentan diferencias significativas al aplicar IMECH entre control de la cólera manifiesta y la capacidad de control de la cólera contenida.

Para los estudiantes de la ciudad de Lima que participaron en actos de violencia y aquellos sin participación, se encontraron diferencias significativas con relación a la ansiedad rasgo mientras que la ansiedad estado no presentó ninguna diferencia significativa. A la inversa que lo anteriormente descrito la cólera estado se ubicó dentro de diferencias significativas ante la cólera rasgo que no la tuvo. Por otro lado la cólera manifiesta evidencia diferencias significativas en contraposición a la cólera contenida y el control de la cólera contenida evidenciaron diferencias significativas inversamente al control de la cólera manifiesta y de la cólera contenida.

En cuanto a los grupos de estudiantes con y sin participación en actos violentos en la ciudad de Trujillo, se encuentra la existencia de diferencias significativas en las siguientes áreas: cólera estado-cólera rasgo, cólera manifiesta-cólera contenida, así mismo control de la cólera manifiesta y control de la cólera contenida. Por otro lado no hubo diferencias significativas entre la ansiedad estado y ansiedad rasgo.

Los datos antes analizados, refuerzan los conceptos de ansiedad, cólera y hostilidad como estados exacerbados de activación emocional que se ha supuesto constituyen salidas 
conductuales para determinados impulsos: el escape o la evitación, liberarse, etc. (Suinn, 1993). Entre otros, Mira y López (1969) se refería a "el miedo, la ira, el amor y el deber" como emociones innatas ligadas al espíritu de toda persona. En cualquier caso, lo "establecido" dentro de esos y otros conceptos tradicionales es que los estados dc activación juegan un papel causal determinante para la personalidad del individuo, lo cual se completa con la afirmación de que a su vez tales estados son más que nada respuestas a ciertos eventos estimulantes (William James, en 1884, fue al parecer el primero que señaló claramente dicha dependencia). Esto último es importante considerarlo para una adecuada concepción de las relaciones entre emociones e instigaciones externas del tipo de la violencia.

Es evidente que la producción de conducta antisocial por parte de los individuos, especialmente de los jóvenes, se correlaciona altamente con los estados emocionales activadores. Esto independientemente de que los entornas familiar y social disfuncionales sean las causas primarias (Kazdin, 1988; Kazdin y Buela-Casal, 1994). Por ello es que los más calificados programas de tratamiento referidos al problema de la violencia humana incluyen módulos para la reducción de la cólera y la ansiedad en poblaciones altamente expuestas al riesgo antisocial (Nóvaco, 1975; Feindler, 1984; Goldstein y Keller, 1991). Sin embargo, no parecen haber estudios explícitamente diseñados para establecer semejante correlación, quizá por su mismo sobreentendimiento. Con el aumento del interés por tales problemas es de esperar que dichos estudios se realicen, como lo pide el sentido común.

\section{CONCLUSIONES}

Como conclusión general del estudio, puede afirmarse que existe una relación baja entre la Ansiedad y la Cólera en estudiantes con y sin participación en actos violentos de Lima y Trujillo. De la misma manera está relación se expresa en los estudiantes que participan y no participan en actos violentos por separado en las ciudades de Lima y Trujillo.

Con respecto a las condiciones emocionales y las disposiciones conductuales adquiridas las respuestas tanto de los alumnos con y sin participación en actos violentos de Lima como de Trujillo no son tan evidentes como las reacciones subjetivas de cólera y rabia en sus dos expresiones (cólera estado-cólera rasgo). Sin embargo en la ciudad de Lima tanto los alumnos que participan o no participan en actos violentos desarrollan estados de ansiedad rasgo implicando esto las diferencias entre las personas en la disposición para responder a situaciones de tensión con cantidades variables de ansiedadestado, siendo esta manifestación mayor en los alumnos que no participan en actos violentos, debido probablemente a las características hábiles de personalidad, y a la sobre exposición de estímulos de acciones violentas, que no se evidencian en la ciudad de Trujillo.

En cuanto a la cólera en sus dos expresiones (cólera-estado y cólera rasgo), encontramos que los alumnos con y sin participación en actos violentos en las dos ciudades muestran diferencias en los sentimientos subjetivos $(C E)$, no así en los estudiantes de Lima con respecto a los de Trujillo que presentan sus expresiones correspondientes $(C R)$. Sin embargo en la ciudad de Lima encontramos que los sentimientos subjetivos de cólera son menores que las expresiones de sus estados emocionales, debido a que los jóvenes alumnos deben aparentar cólera frente a las otras personas como patrón de comportamiento. 
Referente a la cólera manifiesta y contenida. encontramos que los alumnos con y sin participación de Lima y Trujillo evidencian disposición diferencial para el desarrollo de ellas, siendo esta marcada en la ciudad de Trujillo en la que existe una mayor proporción en cuanto a la cólera manifiesta y contenida en los alumnos que participan a actos violentos frente a los que no participan. El mismo fenómeno se presenta en la ciudad de Lima en los grupos de estudiantes con y sin participación en actos violentos con respecto a la cólera manifiesta. La diferencia al comparar ambas ciudades y grupos es que en la ciudad de Trujillo la condición de participación en actos violentos evidencia una mayor cólera contenida y manifiesta.

Respecto al control de la cólera manifiesta y de la cólera contenida por alumnos con y sin participación de las ciudades de Lima y Trujillo, se puede concluir que los alumnos que no participan en actos violentos de la ciudad de Trujillo expresan un mayor control de ellas que los que participan, lo que conduce a una tendencia de estos a ejercer libremente la violencia frente a los otros; de la misma manera pero solo en el control de la cólera contenida se expresa en la ciudad de Lima.

En lo correspondiente a la validez y confiabilidad de los instrumentos, estos fueron válidos. En el caso del IDARE, sus componentes (Ansiedad-Estado, Ansiedad-Rasgo) requirieron de la construcción de subescalas a partir de la aplicación de un análisis factorial.

\section{BIBLIOGRAFIA}

Anicama, J., Mayorga, R., Hinostroza, c., Antinori. b. Ochoa, M. y Tomás, A. (1997). Efectos de un programa cognitivo conductual para prevenir y controlar la violencia de menores en extrema pobreza y alto riesgo. Wiñay Yachay, Revista de la Facultad de Psicología UNFV 2( 1), 71-81.

Anicama, J., Vizcardo, J., Carrasco, J. y Mayorga, E. ( 1999). Estudio epidemiológico sobre la violencia y comportamientos asociados en Lima Metropolitana y Callao. Lima, Perú: MINSAUNFV

Arenas, C. (1998). Los rostros escondidos y la acción social frente a la marginalidad: violencia juvenil de los 90. Revista de psicología de la UNMSM. II (1), 93-106.

Bavermeister, J. (1986). Confiabilidad y validez del Inventario de ansiedad Rasgo-Estado para niños portorriqueños y panameños. Revista Interamericana de Psicología. XX (1-2).

Bending, A. (1962). Factor analytic scales of covert and overt hostility. Journal of consulting Psichology. 26, 200

Comisión Especial del Congreso de la República que investiga las causas y consecuencias de la violencia cotidiana en el país (1998). Iniciativa legislativa. Congreso de la República

Crane, J. (1981). The role of anger, hostility, and aggression in esential hipertension (Doctoral Dissertation University of South Florida, 1981). Dissertation Abstracts Intemational,42, 2982B.

Elizalde, R.; Bulnes, M. Escurra, M. y Dávila, N. (1997). Actitudes hacia la violencia en jóvenes universitarios. Revista de psicología de la UNMSM. 1 (1),107-128.

Feindler, E. (1984). Entrenamiento para el control de la cólera en grupo para jóvenes delincuentes de secundaria. Terapia cognitiva e investigación, 8. 299-311.

García, G. (1983). Ansiedad debilitadora y rendimiento escolar. Revista de Psicología de la PUC. $1(1), 61-65$.

Goldstein, A.P. y Keller, K. (1991). El comportamiento agresivo. Bilbao: Descleé de Brouwer. 
Inga, J. (Setiembre, 1999). Interacciones entre niveles de ansiedad y de autoestima en 1111 grupo de estudiantes de una universidad de Lima [Sumario]. En Colegio de Psicólogos del Perú (Ed.), Memorias del IX Congreso Nacional de Psicología, Lima, Perú: Autor.

Kazdin, A.E. (1988). Tratamiento de la conducta antisocial en la infancia y la adolescencia. Barcelona, España: Martinez Roca.

Kazdin, A.E. y Buela-Casal, G. (1994). Conducta antisocial. Madrid, España: Pirámide.

Mac Gregor, F.E.; Rubio, M. y Vega, R. (1990). Investigación en violencia estructural. Lima.

Masías, e. (1994). Efectos de un programa integral de sustitución de la agresión en un grupo de adolescentes hombres de alto riesgo entre 14 y 18 años de edad, de sectores tugurizados de Barrios Altos. Tesis de Maestría, Lima: UPSMP.

Mira y López, E. (1969). Cuatro gigantes del alma: El miedo, la ira, el amor, el deber. Buenos aires, Argentina: El Ateneo.

Moragues, M.( 1982). Educar para el autogobierno. Lima: Tarea.

Moscoso, M. (1999). Medición de la expresión de la cólera y hostilidad. Revista de la Facultad de psicología de la UNMSM. III(4), 169-188.

Orellana, O. y García , L. (1996). Violencia y representaciones sociales en escolares. Revista

Peruana de psicología. I (1),26-36.

Perales, A. y Sogi, P. (1995). Conductas violentas en adolescentes: Identificación de factores de riesgo para diseño de programa preventivo. Lima: Serie Monografías de Investigación. Instituto Nacional de Salud Mental "Hideyo Noguchi".

Nóvaco, R. (1975). Anger control: The development and evaluation of an experimental treatment. Lexinton, EEUU: Health. And Co.

Quintana, A. (1 998). Estrés y afrontamiento del estrés frente al examen en educación superior: ¿Un estilo o un proceso? Revista de Investigación en Psicología de la UNMSM. ¡(2), 113-133.

Spielberger, CH. (1983). Manual for the state-Trait anger Expression Inventory (STAI). Palo alto: consulting Psychologist Press.

Spielberger, Ch. y Díaz-Guerrero, R. (1975). Inventario de ansiedad estado-rasgo. México: El manual Moderno.

Staats, A. w. (1979). Conductismo social. México D.F., México: el Manual Moderno.

Suinn, R.M. (1993). Entrenamiento en manejo de ansiedad. Bilbao, España: Descleé de Brouwer.

Tong, F. (1995). Los jóvenes pandilleros. Entre el estigma y la epopeya. Lima: Tarea.

Ugarriza, N. (1998). Normalización del Inventario Multicultural de la Expresión de CóleraHostilidad en estudiantes universitarios. Revista de Investigación en Psicología de la UNMSM. I (1),59-88.

Vallejos, J. y Montero, V. (1990). La Psicología comunitaria en el Perú. Psicología actual. III(6), 15-21.

Varona, (1991). Efectos de un programa para la sustitución de la agresión mediante el desarrollo de habilidades sociales y el autocontrol en menores de 11-15 años, en condiciones especialmente difíciles, institucionalizados del COMAIN. Tesis de Maestría, Lima: UPCH.

Velásquez, e. (1999). La violencia juvenil en Lima Metropolitana. Revista de Investigación en Psicología de la UNMSM. Vol. 1,No 1, pp. 91 - 102. 


\section{ANEXO No 1}

ANALISIS DE ITEMS DEL COEFICIENTE DE VALIDEZ ITEM-TEST CORREGIDO DE CADA UNA DE LAS SUB-AREAS DEL IDARE

\begin{tabular}{|c|c|c|c|}
\hline \multicolumn{4}{|c|}{ ANSIEDAD ESTADO } \\
\hline Item & $\mathbf{r}$ & Item & $\mathbf{r}$ \\
\hline 1 &, 13 & 11 &, 26 \\
2 &, 15 & 12 &, 16 \\
3 &, 16 & 13 &, 21 \\
4 &, 14 & 14 &, 15 \\
5 &, 30 & 15 &, 22 \\
6 &, 17 & 16 &, 31 \\
7 &, 11 & 17 &, 11 \\
8 &, 23 & 18 &, 16 \\
9 &, 24 & 19 &, 31 \\
10 &, 29 & 20 &, 27 \\
\hline
\end{tabular}

\begin{tabular}{|c|c|c|c|}
\hline \multicolumn{4}{|c|}{ ANSIEDAD RASGO } \\
\hline Item & $\mathbf{r}$ & Item & $\mathbf{r}$ \\
\hline 21 &,- 02 & 31 &, 27 \\
22 &, 18 & 32 &, 20 \\
23 &, 23 & 33 &,- 14 \\
24 &, 21 & 34 &, 16 \\
25 &, 22 & 35 &, 27 \\
26 &, 03 & 36 &, 01 \\
27 &, 02 & 37 &, 25 \\
28 &, 26 & 38 &, 29 \\
29 &, 28 & 39 &, 04 \\
30 &,- 05 & 40 &, 30 \\
\hline \multicolumn{4}{|c}{}
\end{tabular}

ANEXO No 2

CONFIABILIDAD POR CONSISTENCIA DE VALIDEZ ITEM-TEST COEFICIENTE ALFA DE CROMBACH

\begin{tabular}{|c|c|}
\hline AREAS DEL IDARE & Coeficiente de Correlación \\
\hline ANSIEDAD ESTADO & .6033 \\
ANSIEDAD RASGO & .5029 \\
\hline
\end{tabular}

\section{ANALISIS DE ITEMS A TRAVÉZ DEL COEFICIENTE DE VALIDEZ ITEN- TEST CORREGIDO DE CADA UNA DE LAS SUB-AREAS DEL IMECH COLERA ESTADO COLERA RASGO}

\begin{tabular}{|c|c|c|c|}
\hline \multicolumn{4}{|c|}{ ANSIEDAD ESTADO } \\
\hline Item & $\mathbf{r}$ & Item & $\mathbf{r}$ \\
\hline 1 & 0.1 & 11 & 0.26 \\
1 & 0.6 & 6 & 0.73 \\
2 & 0.6 & 7 & 0.72 \\
3 & 0.5 & 8 & 0.73 \\
4 & 0.7 & 9 & 0.67 \\
5 & 0.7 & 10 & 0.68 \\
\hline
\end{tabular}

\begin{tabular}{|c|c|c|c|}
\hline \multicolumn{4}{|c|}{ COLERA RASGO } \\
\hline Item & r & Item & r \\
\hline 21 & -0.2 & 31 &, 27 \\
1 &, 47 & 6 &, 49 \\
2 &, 49 & 7 &, 46 \\
3 &, 57 & 8 &, 44 \\
4 &, 49 & 9 &, 47 \\
5 &, 58 & 10 &, 57 \\
\hline
\end{tabular}

\begin{tabular}{|c|c|c|c|}
\hline \multicolumn{2}{|c|}{$\begin{array}{c}\text { CÓLERA } \\
\text { MANIFIESTA }\end{array}$} & \multicolumn{2}{|c|}{$\begin{array}{c}\text { CÓLERA } \\
\text { CONTENIDA }\end{array}$} \\
\hline Item & $\mathbf{r}$ & Item & $\mathbf{r}$ \\
\hline 2 & ,39 & 3 &, 35 \\
\hline 6 & ,43 & 4 &, 35 \\
\hline 8 &, 44 & 9 &, 22 \\
\hline 14 & ,39 & 11 & ,23 \\
\hline 15 & ,13 & 2 & ,38 \\
\hline 23 & ,21 & 13 & ,42 \\
\hline
\end{tabular}

\begin{tabular}{|c|c|c|c|}
\hline \multicolumn{2}{|c|}{ CONTROL } & \multicolumn{2}{c|}{ CONTROL C. } \\
MANTENIDA. & \multicolumn{2}{|c|}{ CONTENIDA } \\
\hline Item & r & Item & r \\
\hline 1 &, 46 & 17 &, 46 \\
5 &, 49 & 18 &, 54 \\
7 &, 45 & 19 &, 57 \\
10 &, 41 & 20 &, 51 \\
16 &, 48 & 21 &, 54 \\
24 &, 51 & 22 &, 37 \\
\hline
\end{tabular}




\section{ANEXO No 4 \\ CONFIABILIDAD DEL IMECH POR CONSISTENCIA INTERNA A TRAVÉZ DEL COEFICIENTE ALFA DE CROMBACH}

\begin{tabular}{|l|c|}
\hline \multicolumn{1}{|c|}{ AREAS DEL IMECH } & Coeficiente de Correlación \\
\hline Cólera Estado &, 9005 \\
Cólera Rasgo &, 8191 \\
Cólera Manifiesta &, 5937 \\
Cólera Contenida &, 5845 \\
Control de la Cólera Manifiesta &, 7334 \\
Control de la Cólera Contenida &, 7569 \\
\hline
\end{tabular}

\section{ANEXO No 5 \\ BAREMOS DE LOS ALUMNOS SIN PARTICIPACIÓN EN ACTOS VIOLENTOS DE LA CIUDAD E LIMA n = 556}

\begin{tabular}{|c|cccccccc|c|}
\hline \multirow{2}{*}{$\mathbf{P P}$} & \multicolumn{7}{|c|}{ PD } & \multirow{2}{*}{ PP } \\
\cline { 2 - 9 } & AE & AR & CE & CR & CM & CC & CCM & CCC & \\
\hline 99 & 57 & 63 & 33 & 38 & 18 & 21 & 24 & 24 & 99 \\
95 & 53 & 58 & 25 & 32 & 16 & 18 & 23 & 23 & 95 \\
90 & 51 & 55 & 21 & 30 & 15 & 16 & 22 & 22 & 90 \\
85 & 49 & 54 & 17 & 27 & 14 & 15 & 21 & 20 & 85 \\
80 & 48 & 52 & 16 & 26 & 13 & 14 & 20 & 19 & 80 \\
\hline 75 & 47 & 51 & 15 & 25 & 13 & 13 & 19 & 18 & 75 \\
70 & 46 & 50 & 13 & 24 & 12 & 13 & 18 & 18 & 70 \\
65 & 45 & 49 & 13 & 23 & 11 & 12 & 18 & 17 & 65 \\
60 & 45 & 48 & 12 & 22 & 11 & 12 & 17 & 16 & 60 \\
55 & 44 & 48 & 12 & 21 & 11 & 12 & 17 & 16 & 55 \\
\hline 50 & 43 & 47 & 11 & 20 & 10 & 11 & 16 & 15 & 50 \\
\hline 45 & 42 & 46 & 11 & 20 & 10 & 11 & 15 & 15 & 45 \\
40 & 42 & 45 & 11 & 19 & 10 & 10 & 15 & 14 & 40 \\
35 & 41 & 45 & 10 & 18 & 9 & 10 & 14 & 14 & 35 \\
30 & 40 & 44 & 10 & 17 & 9 & 9 & 14 & 13 & 30 \\
25 & 39 & 43 & 10 & 16 & 9 & 9 & 13 & 12 & 25 \\
\hline 20 & 38 & 42 & 10 & 16 & 8 & 8 & 12 & 12 & 20 \\
15 & 36 & 41 & 10 & 15 & 8 & 8 & 12 & 11 & 15 \\
10 & 35 & 40 & 10 & 14 & 7 & 7 & 11 & 11 & 10 \\
5 & 33 & 38 & 10 & 13 & 6 & 6 & 10 & 10 & 5 \\
1 & 31 & 34 & 10 & 10 & 6 & 6 & 7 & 8 & 1 \\
\hline PP & AE & AR & CE & CR & CM & $\mathbf{C C}$ & $\mathbf{C C M}$ & $\mathbf{C C C}$ & $\mathbf{P P}$ \\
\hline
\end{tabular}




\section{ANEXO No 6 \\ BAREMOS DE LOS ALUMNOS CON PARTICIPACIÓN EN ACTOS VIOLENTOS DE LA CIUDAD DE LIMA n = 516}

\begin{tabular}{|c|ccccccccc|c|}
\hline \multirow{2}{*}{ PP } & \multicolumn{7}{|c|}{ PD } & \multirow{2}{*}{ PP } \\
\cline { 2 - 8 } & $\mathbf{A E}$ & $\mathbf{A R}$ & $\mathbf{C E}$ & $\mathbf{C R}$ & $\mathbf{C M}$ & $\mathbf{C C}$ & $\mathbf{C C M}$ & $\mathbf{C C C}$ & \\
\hline 99 & 58 & 62 & 34 & 38 & 19 & 21 & 24 & 24 & 99 \\
95 & 52 & 58 & 26 & 33 & 17 & 18 & 23 & 22 & 95 \\
90 & 52 & 54 & 23 & 30 & 16 & 16 & 22 & 21 & 90 \\
85 & 49 & 52 & 21 & 27 & 15 & 15 & 20 & 20 & 85 \\
80 & 48 & 51 & 18 & 26 & 14 & 14 & 19 & 18 & 80 \\
\hline 75 & 47 & 50 & 17 & 25 & 13 & 14 & 18 & 18 & 75 \\
70 & 46 & 49 & 15 & 24 & 12 & 13 & 18 & 17 & 70 \\
65 & 45 & 49 & 14 & 23 & 12 & 12 & 17 & 16 & 65 \\
60 & 44 & 48 & 13 & 23 & 12 & 12 & 17 & 16 & 60 \\
55 & 44 & 47 & 13 & 22 & 11 & 11 & 16 & 15 & 55 \\
\hline 50 & 43 & 46 & 12 & 21 & 11 & 10 & 15 & 15 & 50 \\
\hline 45 & 42 & 46 & 11 & 20 & 10 & 10 & 14 & 14 & 45 \\
40 & 41 & 45 & 11 & 19 & 10 & 10 & 14 & 14 & 40 \\
35 & 41 & 44 & 11 & 18 & 10 & 10 & 13 & 13 & 35 \\
30 & 40 & 43 & 10 & 17 & 9 & 9 & 12 & 12 & 30 \\
25 & 39 & 42 & 10 & 17 & 9 & 9 & 12 & 12 & 25 \\
\hline 20 & 38 & 41 & 10 & 16 & 8 & 8 & 12 & 12 & 20 \\
15 & 37 & 40 & 10 & 15 & 8 & 8 & 11 & 11 & 15 \\
10 & 36 & 39 & 10 & 14 & 7 & 7 & 11 & 10 & 10 \\
5 & 34 & 36 & 10 & 13 & 7 & 7 & 9 & 9 & 5 \\
1 & 30 & 31 & 10 & 11 & 6 & 6 & 6 & 7 & 1 \\
\hline PP & $\mathbf{A E}$ & $\mathbf{A R}$ & $\mathbf{C E}$ & $\mathbf{C R}$ & $\mathbf{C M}$ & $\mathbf{C C}$ & $\mathbf{C C M}$ & $\mathbf{C C C}$ & $\mathbf{P P}$ \\
\hline & & & & & & & & & \\
\hline
\end{tabular}




\section{ANEXO No 7 \\ BAREMOS DE LOS ALUMNOS SIN PARTICIPACIÓN EN ACTOS VIOLENTOS DE LA CIUDAD DE TRUJILLO $\mathbf{n}=\mathbf{2 2 3}$}

\begin{tabular}{|c|ccccccccc|c|}
\hline \multirow{2}{*}{ PP } & \multicolumn{7}{|c|}{ PD } & \multirow{2}{*}{ PP } \\
\cline { 2 - 9 } & $\mathbf{A E}$ & $\mathbf{A R}$ & $\mathbf{C E}$ & $\mathbf{C R}$ & $\mathbf{C M}$ & $\mathbf{C C}$ & $\mathbf{C C M}$ & $\mathbf{C C C}$ & \\
\hline 99 & 56 & 62 & 30 & 38 & 22 & 21 & 24 & 24 & 99 \\
95 & 52 & 53 & 24 & 33 & 18 & 18 & 24 & 23 & 95 \\
90 & 50 & 55 & 18 & 29 & 17 & 16 & 22 & 22 & 90 \\
85 & 49 & 52 & 15 & 28 & 15 & 15 & 21 & 21 & 85 \\
80 & 48 & 52 & 14 & 26 & 14 & 14 & 20 & 20 & 80 \\
\hline 75 & 47 & 51 & 13 & 25 & 13 & 13 & 20 & 19 & 75 \\
70 & 46 & 50 & 12 & 24 & 12 & 12 & 19 & 18 & 70 \\
65 & 45 & 49 & 12 & 23 & 11 & 12 & 18 & 18 & 65 \\
60 & 44 & 48 & 11 & 22 & 11 & 12 & 18 & 18 & 60 \\
55 & 44 & 48 & 11 & 21 & 11 & 11 & 18 & 17 & 55 \\
\hline 50 & 43 & 47 & 11 & 20 & 10 & 11 & 17 & 16 & 50 \\
\hline 45 & 42 & 46 & 10 & 19 & 10 & 10 & 17 & 16 & 45 \\
40 & 42 & 46 & 10 & 18 & 10 & 10 & 16 & 15 & 40 \\
35 & 41 & 45 & 10 & 18 & 9 & 10 & 15 & 14 & 35 \\
30 & 41 & 44 & 10 & 16 & 9 & 9 & 14 & 14 & 30 \\
25 & 40 & 44 & 10 & 16 & 9 & 9 & 14 & 13 & 25 \\
\hline 20 & 39 & 43 & 10 & 15 & 8 & 9 & 13 & 12 & 20 \\
15 & 38 & 42 & 10 & 14 & 7 & 8 & 12 & 12 & 15 \\
10 & 36 & 41 & 10 & 13 & 7 & 7 & 11 & 11 & 10 \\
5 & 34 & 39 & 10 & 12 & 6 & 7 & 10 & 10 & 5 \\
1 & 30 & 34 & 10 & 10 & 6 & 6 & 9 & 6 & 1 \\
\hline PP & $\mathbf{A E}$ & $\mathbf{A R}$ & $\mathbf{C E}$ & $\mathbf{C R}$ & $\mathbf{C M}$ & $\mathbf{C C}$ & $\mathbf{C C M}$ & $\mathbf{C C C}$ & $\mathbf{P P}$ \\
\hline
\end{tabular}




\section{ANEXO No 8 \\ BAREMOS DE LOS ALUMNOS CON PARTICIPACIÓN EN ACTOS VIOLENTOS DE LA CIUDAD DE TRUJILLO $\mathrm{n}=215$}

\begin{tabular}{|c|ccccccccc|c|}
\hline \multirow{2}{*}{$\mathbf{P P}$} & \multicolumn{7}{|c|}{ PD } & \multirow{2}{*}{ PP } \\
\cline { 2 - 9 } & $\mathbf{A E}$ & AR & CE & CR & CM & CC & CCM & CCC & \\
\hline 99 & 59 & 61 & 33 & 40 & 21 & 20 & 24 & 24 & 99 \\
95 & 54 & 56 & 26 & 34 & 18 & 18 & 21 & 22 & 95 \\
90 & 51 & 55 & 23 & 32 & 17 & 17 & 20 & 20 & 90 \\
85 & 50 & 56 & 20 & 30 & 16 & 16 & 19 & 19 & 85 \\
80 & 49 & 56 & 19 & 29 & 15 & 15 & 18 & 18 & 80 \\
\hline 75 & 47 & 52 & 17 & 28 & 14 & 14 & 18 & 17 & 75 \\
70 & 46 & 51 & 16 & 27 & 14 & 14 & 18 & 17 & 70 \\
65 & 45 & 50 & 15 & 25 & 12 & 13 & 17 & 16 & 65 \\
60 & 45 & 49 & 14 & 25 & 12 & 13 & 16 & 16 & 60 \\
55 & 444 & 48 & 14 & 23 & 11 & 12 & 16 & 15 & 55 \\
\hline 50 & 43 & 47 & 13 & 23 & 11 & 12 & 15 & 14 & 50 \\
\hline 45 & 43 & 47 & 12 & 22 & 11 & 11 & 14 & 14 & 45 \\
40 & 42 & 46 & 12 & 21 & 10 & 11 & 13 & 14 & 40 \\
35 & 41 & 45 & 11 & 20 & 10 & 11 & 13 & 13 & 35 \\
30 & 40 & 45 & 11 & 20 & 10 & 10 & 12 & 12 & 30 \\
25 & 39 & 44 & 11 & 19 & 9 & 10 & 12 & 12 & 25 \\
\hline 20 & 38 & 43 & 10 & 18 & 9 & 9 & 11 & 12 & 20 \\
15 & 37 & 42 & 10 & 16 & 8 & 9 & 10 & 11 & 15 \\
10 & 36 & 41 & 10 & 16 & 8 & 8 & 9 & 10 & 10 \\
5 & 34 & 38 & 10 & 14 & 7 & 7 & 8 & 9 & 5 \\
1 & 30 & 36 & 10 & 10 & 6 & 6 & 6 & 7 & 1 \\
\hline PP & AE & AR & CE & CR & CM & CC & CCM & CCC & PP \\
\hline
\end{tabular}

